\title{
LA GESTIÓN UNIVERSITARIA FRENTE A LOS ACTUALES DESAFÍOS
}

\section{Introducción}

Las instituciones de la educación superior son parte del entorno social y, por ende, reflejan las tendencias predominantes del momento histórico o de la época que les toca vivir. La universidad de la Edad Media se fundaba en un modelo educativo centrado fundamentalmente en la enseñanza de las disciplinas llamadas "clásicas", como la filosofía y la teología; estaba orientada a formar a una elite y los estudios tenían una finalidad en sí misma. Vistas desde la perspectiva de su organización interna, las universidades recibían aportes de benefactores privados o de la propia Iglesia y tenían un gobierno ejercido por los propios scholars. Un esquema que hoy calificaríamos de precario: una estructura de carácter horizontal y un modelo de decisiones descentralizado donde la responsabilidad era compartida por los pares.

Este modelo, en cierta forma de claustro, evoluciona varios siglos más tarde hacia la apertura social y el mayor contacto con el medio. La influencia de la revolución industrial y el desarrollo científico y la creación de los estados nacionales son la antesala de un modelo no sólo más universal en lo disciplinario, sino, además, origen de instituciones mucho más inmersas en la sociedad, de cara a las crecientes y múltiples necesidades de la comunidad.

Ello queda de manifiesto de un modo muy elocuente en los escritos de John Henry Newman ${ }^{1}$, a mediados del siglo XIX, quien declara que el arte de la universidad "es el arte de la vida social, y su fin es armonizar con el mundo". El objetivo social de la universidad

1 Newman, J.H. (1996) The idea of a university. Frank Turner Editor, Yale University Press. 
es formar good members of society. Pero la idea de universidad estaba en esos años aun lejos del modelo en su versión contemporánea. Desde luego, porque, aunque se reconocía el papel de la investigación, Newman no la visualizaba como un motor para generar nuevo conocimiento útil, pese a ser una época donde tomaba fuerza el enfoque utilitarista. Asimismo, es posible que la propia experiencia de gestión de Newman, al fundar en Dublín una nueva casa de estudios superiores, estuviera todavía lejos de lo que hoy se asocia a una administración universitaria moderna. En términos de Frank T. Turner², "la palabra universidad sigue siendo la misma hoy como lo era su uso en los años de Newman; sin embargo, no lo es en términos de la realidad que describe [...] Muchos administradores universitarios continúan hablando de las universidades como los lugares para buscar el conocimiento per se, pero la realidad que ellos deben administrar es bastante diferente. Las universidades constituyen hoy uno de los principales vehículos para conseguir conocimiento útil y rentable".

Pero el tránsito desde aquel paradigma de universidad al modelo actual no ha sido lineal, sino sujeto a saltos discretos, en especial con cambios radicales en las últimas dos décadas. No sólo porque en el presente se les pide a las universidades que jueguen un papel más determinante en el desarrollo social, político y económico de los países, sino también porque su existencia ha dejado de ser fácil y se ven enfrentadas a un ambiente más hostil. Entre los aspectos claves en este nuevo marco están los fenómenos de la competencia y el financiamiento, que las obligan a revisar sus procesos internos, incluso como condición de supervivencia en el largo plazo. Esto es particularmente válido en EE.UU., donde la idea predominante es el radical cambio desde una visión de universidad como una institución eminentemente social, hacia otra que la concibe como parte de una industria estructurada en torno a entidades corporativas que compiten en el mercado, que deben autofinanciarse y que producen una amplia gama de bienes y

2 Turner, F.M. Editor de The idea of a university, p. 282. 
servicios. En ese contexto, las instituciones universitarias están llamadas a ser lo que Zemsky ${ }^{3}$ denomina mission-centered and market smart.

El desarrollo universitario en Chile no ha estado ajeno a estos cambios y guarda alguna similitud, si bien a otra escala, con lo que observamos en el hemisferio norte y, en especial, en EE.UU. Las universidades se ven compelidas a realizar una gestión que les permita permanecer fieles a su misión fundacional, pero disponiendo, a la vez, de la soltura y diligencia adecuadas para reaccionar con eficacia y eficiencia frente a los nuevos desafíos.

En este artículo se analizan las transformaciones en la gestión de las universidades, teniendo en perspectiva a un tipo de universidad complejo, sin fines de lucro, de vocación pública y con acento en la investigación. Si bien hay muchos elementos comunes en el diseño y organización de las universidades, fácilmente identificables, hay matices que ponen diferencias importantes. Uno de ellos es el lucro como un factor a considerar en su manejo. Así, en la primera parte se aborda este tema como un elemento relevante para no perder de vista al momento de definir el modelo de gestión universitaria. Luego se describen dos procesos que son esencialmente transversales a toda organización: la planificación estratégica que traza las grandes líneas del desarrollo institucional y el tema del gobierno universitario, es decir, la organización interna que permite dar el cauce apropiado a dicho plan.

La segunda parte del texto estudia las reacciones de la universidad frente a las nuevas tendencias y desafíos, las que, en lo sustantivo, condicionan la gestión con miras a entregar respuestas adecuadas frente a los requerimientos de un entorno diverso y cambiante.

3 Zemsky, R. (1999) The third imperative, Policy Perspectives, $9 \mathrm{~N}^{\circ} 1$. 


\section{La organización interna}

\section{Las universidades y el lucro}

Históricamente las universidades en el mundo entero se organizaron como instituciones sin fines de lucro, como reflejo del fenómeno que en economía se conoce como "asimetrías de información"4. En las universidades se cumple estrictamente el supuesto de información asimétrica, porque es evidente que los estudiantes, o sea los que demandan educación, no cuentan con todos los antecedentes para una elección que no tenga un grado importante de incertidumbre. En ese contexto, la inversión en reputación -y el no lucro como modelo de gestión- es crucial para que las universidades puedan neutralizar la desconfianza del alumno o de su padre por la carencia de información.

Aquí el concepto de lucro no niega la posibilidad de que las universidades puedan eventualmente generar excedentes. El énfasis en el "no lucro" está puesto más bien en que en tales instituciones no hay que rendir cuentas a terceros, digamos a los dueños del capital, como en la visión clásica de empresa donde los dueños son los accionistas, quienes exigirán una rentabilidad por la inversión como ocurre en el mundo empresarial corporativo.

Es cierto, sin embargo, que las universidades con estas características suelen organizarse en torno a consejos compuestos por personas de prestigio que garantizan la validez y seriedad del proyecto educativo. Con todo, si el lucro está presente como un objetivo central de la operación de una universidad, el foco de la

4 Según este concepto, cuando no hay información para una de las partes que interviene en un mercado ésta queda muy vulnerable frente a comportamientos oportunistas de su contraparte, siendo prácticamente imposible suscribir contratos que garanticen al detalle que no será defraudada en su relación de intercambio. En dichos mercados la creación de lazos de confianza reemplaza a los contratos tradicionales, porque la confianza es la garantía de seriedad. Ahora bien, la mayor fortaleza para construirla y que sea creíble es la adopción de un modelo de administración sin fin de lucro, porque eso fortalece la percepción de que la empresa que ofrece algo no tendrá otro objetivo que responder a la promesa de un producto con la calidad esperada. 
gestión no es el mismo: la selección y aprobación de proyectos debe pasar por el filtro de su rentabilidad económica y la orientación cambia. Más aún, la evidencia señala que la educación superior se ha convertido en una actividad muy rentable, lo cual queda de manifiesto por el creciente flujo de inversiones privadas, tanto en Chile como en Estados Unidos ${ }^{5}$. En este último país, la rentabilidad de las inversiones en el ámbito educacional ha sido el doble respecto de un índice promedio de mercado ${ }^{6}$. El caso más exitoso de rentabilidad es el de la Universidad de Phoenix, la mayor inversión privada en educación superior, que cuenta con 60.000 alumnos, se orienta a la docencia, tiene sólo personal part-time y un fuerte componente de educación a distancia de bajo costo ${ }^{7}$.

\section{El plan estratégico y el presupuesto}

Las transformaciones en la gestión de las instituciones de educación superior son consecuencia directa de los nuevos desafíos que hoy enfrenta el mundo universitario. No hay precedentes en su historia en que hayan estado sometidas a un grado de tensión con la fuerza y frecuencia que se da en la actualidad, donde no hay espacios para improvisar respuestas, es necesario saber cómo reaccionar y la planificación es crucial para fijar la carta de navegación futura. El plan estratégico es el primer instrumento de gestión. Las universidades deben construir un plan de desarrollo que enmarque la vida académica y sirva de referente básico para que la comunidad universitaria sepa cuáles son las principales aspiraciones y políticas en el marco de su misión fundacional y en la promoción de los valores institucionales. En este proceso es decisivo el diagnóstico inicial, que se logra con un análisis FODA clásico y que permite

5 En el caso chileno, la ley sólo admite instituciones de educación superior sin fines de lucro, lo cual crea una situación poco deseable, ya que es evidente que hay privados que han invertido en esta industria con miras a obtener rentabilidades, lo que se traduce en poca transparencia y amenazas de regulaciones excesivas por parte de una autoridad recelosa.

6 Collis, D.J. (2000). When industries change, revisited: new scenarios for higher education, (paper no publicado).

7 Ibíd. 
conocer las fortalezas y debilidades, las oportunidades del entorno y las amenazas.

El plan de desarrollo debe tener un horizonte de largo plazo como única manera de pensar la universidad del futuro, es decir, proyectándose a varios años y elaborando un conjunto coherente de políticas que permita el logro de los objetivos estratégicos. Los planes anuales específicos definidos para las distintas unidades de la universidad sirven para asignar tareas y responsabilidades y, luego, para monitorear los avances en las diferentes áreas.

La eficacia de un plan se mide por su capacidad para encauzar las fuerzas académicas, de modo de alcanzar los objetivos propuestos en los plazos convenidos. La eficiencia de éste, sin embargo, está en directa relación con su capacidad para alcanzar las metas con el menor costo posible en recursos económicos. En consecuencia, la asociación entre recursos y objetivos debe ser una tarea permanente para lo cual el empleo del análisis costobeneficio es una herramienta útil e irreemplazable. Definido ya el plan estratégico y evaluadas económicamente las políticas y acciones específicas, se construye entonces el presupuesto de gastos para proceder a la asignación de recursos. Las universidades que proceden según esta secuencia centralizan la captación de los recursos para el financiamiento sin perjuicio de otorgar libertad a las unidades académicas para la generación de fuentes propias y libertad para gastarlos. Las ventajas de este modelo son una mayor garantía sobre el logro de los objetivos del plan y un mayor control sobre los gastos. La generación descentralizada del total de ingresos y la completa autonomía en el gasto no es recomendable. Desde luego, exige el uso de precios de transferencias entre las unidades-por ejemplo, para la docencia-o de instalaciones físicas, y fuerza a negociaciones improductivas con los riesgos obvios de deteriorar relaciones y afectar los planes académicos ${ }^{8}$.

8 Ehrenberg, Ronald (2000). Tuition rising: why college costs so much, Harvard University Press. 


\section{El gobierno de la universidad}

Es ésta una de las pocas organizaciones sociales donde la idea de gobierno compartido es condición necesaria para el éxito institucional, pero también puede ser un serio obstáculo para su desarrollo, en tanto no exista un adecuado diseño en este balance de poderes. Esto es especialmente relevante en la actualidad, donde la multiplicidad de fines, áreas y tareas estratégicas -en un contexto de escasez de recursos- obliga a una gestión donde exista una efectiva división del trabajo en función de competencias específicas. Por cierto, los modelos organizativos admiten una diversidad de esquemas; sin embargo, la idea de "buen gobierno" se asocia al concepto de compartir el poder. Hablar de gobierno compartido alude al concepto de cooperación entre las partes. En el mundo anglosajón se expresa a través del término shared governance, es decir, el buen ejercicio del poder en un contexto participativo en las decisiones.

El gobierno compartido delega en las unidades las decisiones sobre selección, promoción y retiro del personal académico, así como aquellas sobre el desarrollo de los currículos, el contenido temático en la enseñanza, las líneas de investigación y el otorgamiento de grados. Las decisiones sobre la elaboración y conducción de los planes estratégicos de corto y mediano plazo, la generación de recursos, la planificación de los gastos, el manejo presupuestario y financiero y la inversión en equipamiento e infraestructura se radican en la dirección central. Un tercer pilar en este esquema es la presencia de un organismo colegiado, consejo superior o senado académico, instancia legislativa de discusión y debate que enriquece el proceso de toma de decisiones y sirve de generoso apoyo para la gestión. En esta instancia de carácter reflexivo hay cabida para los estudiantes, pero en un rol de consejería y no de toma de decisiones. Ellos tienen mucho que aportar, particularmente en lo concerniente a las definiciones curriculares y actividades extraprogramáticas; sin embargo, no se recomienda que su participación se extienda a otras áreas y, menos 
todavía, que tengan poder en las estructuras de gobierno. En otras partes existen también consejos consultivos (board of trustees) compuestos por personas de prestigio y de conocida trayectoria profesional de la sociedad, y, en algunos casos, por quienes contribuyen también al financiamiento de la universidad.

Los desafíos que se plantean a la universidad contemporánea requieren el concurso de múltiples áreas; además, los procesos son transversales y envuelven normalmente una gran complejidad. Uno de los problemas mayores tiene que ver con la relación entre la autoridad superior y las facultades. La energía creadora de los académicos debe encauzarse hacia la generación de contenidos, pero sus habilidades no están en general en la gestión estratégica. La tradición académica del debate reflexivo y de la búsqueda del consenso son características poco apropiadas para una gestión que se realiza en un contexto de gran dinamismo. Hay también una cultura muy arraigada que, típicamente, se sostiene en ciertos valores como la autonomía y la libertad académicas, y que muchas veces entra en conflicto con las exigencias de responsabilidad, el cumplimiento de los plazos y compromisos, y los requisitos de accountability, que no siempre tienen una recepción adecuada en el mundo académico. A diferencia de una empresa tradicional, en las universidades el tenure, mediante el cual el profesor se incorpora de hecho a la universidad y no puede ser removido, sino por razones muy de peso, establece una suerte de inamovilidad que consolida su poder interno y puede, a veces, impedir una gestión eficiente. Con todo, la responsabilidad que le cabe al mundo académico en la conducción de la universidad, en su propio campo de competencia, no lo exime de ejercer una gestión ágil y eficiente.

En suma, la idea de gobierno compartido supone un balance de poderes que alude a una separación de roles y competencias, junto con un diseño institucional que establece instancias de coordinación a través de un organismo colegiado donde se legisla y se toman las decisiones más relevantes. 


\section{Los nuevos desafíos y el mundo de la gestión \\ El entorno de la competencia ${ }^{9}$}

En la enseñanza del pregrado, especialmente, la competencia de nuevos oferentes de cursos y programas amenaza erosionar lo que hasta hace pocos años eran barreras infranqueables que protegían a la universidad tradicional. La entrada de nuevos rivales se ha hecho más expedita por la dramática caída en los costos, originada en los avances tecnológicos de Internet y la educación a distancia. Esto mismo ha ampliado las opciones de formación desde otras fuentes, o sea, la presencia de nuevos sustitutos respecto de la oferta de enseñanza tradicional. Los mismos productos complementarios a la educación, como los computadores, no tienen en las universidades la única fuente de demanda lo que debilita su posición compradora. Por otra parte, el estamento académico cuenta con mayor poder de mercado al abrirse las alternativas laborales y los clientes -estudiantestienen mayores posibilidades de aprendizaje por lo cual son más sensibles a alzas de aranceles, lo que incrementa a su vez su poder de mercado.

La respuesta a este medio más amenazante no consiste en soluciones mágicas, sino en un refuerzo de las fortalezas competitivas en las áreas tradicionales, una revisión de lo que puede externalizarse para una mayor eficiencia productiva y, desde luego, evaluar las alternativas de competencia en los mercados emergentes.

Una herramienta importante es diferenciarse de los competidores mediante una enseñanza presencial de calidad, lo que requiere invertir en personal académico, equipamiento, infraestructura, entre otros. También es una oportunidad aprovechar el peso de la trayectoria y la creación de lazos filiales con sus ex alumnos, para llegar primeros a un mercado incipiente, pero que crece: la educación para toda la vida. Otro camino de exploración es revisar la cadena productiva. Hacerse preguntas pertinentes

9 Collis, D.J. When industries change: scenarios for higher education (paper no publicado). 
como: ¿se justifica contar con una biblioteca al estilo clásico en un medio donde el acceso electrónico ofrece opciones de calidad y bajo costo? ¿Por qué no externalizar los servicios de aseo, vigilancia, alimentación, entre otros?

Por último, las universidades no debieran marginarse a priori de competir en otros campos como la educación a distancia, por ejemplo, expandiendo allí sus dominios y creando nuevos liderazgos y lealtades. No obstante, hay que precaverse, al mismo tiempo, de no descuidar los productos tradicionales y resguardar la calidad de lo que se ofrece en los nuevos mercados para no arriesgar el prestigio institucional.

\section{Qué se enseña y cómo se enseña en el pregrado}

Uno de los fenómenos más característicos de los nuevos tiempos es la necesaria y profunda reforma que demandan los programas de pregrado de las universidades ${ }^{10}$. Ella se encamina a flexibilizar los currículos, ampliar la formación general y permitir, por esta vía, que los estudiantes adquieran no sólo las competencias específicas, sino que, además, sean capaces de tener una visión amplia del mundo, aspecto decisivo para adaptarse a un medio en continuo cambio. Por otra parte, estas transformaciones obligan a fortalecer la gestión académica, de manera tal de contar con estimaciones sólidas con las cuales proyectar los efectos sobre la demanda por docencia y el impacto consecuente en los recursos de estos cambios curriculares.

Asimismo, en forma cada vez más acentuada las universidades están apoyando el aspecto formativo de la docencia. Esto que parece obvio y sencillo no lo es, salvo que por formación se entienda sólo la instrucción o el simple desarrollo de los contenidos y no se repare en, al menos, dos elementos esenciales de un aprendizaje efectivo: la metodología de la enseñanza y la empatía del profesor. Las

10 Es la tendencia predominante en Europa después del Acuerdo de Bolonia, que fija plazos para reformas curriculares, las cuales, en esencia, convergen hacia el modelo americano. 
universidades no pueden soslayar una labor irrenunciable cual es la de formar personas, estimular el desarrollo cultural y fomentar valores; esa es tarea de profesores, o sea, de maestros idóneos para tales fines. Estas habilidades pedagógicas pueden ser desarrolladas por medio de talleres y cursos especiales, lo que requiere, por cierto, capacidad de liderazgo y poder de convicción de las autoridades superiores para persuadir a los docentes sobre la conveniencia de estos aprendizajes.

\section{Adaptación al impacto de las nuevas tecnologías de información}

La revolución que ha significado la irrupción de las nuevas tecnologías de información plantea un dilema, en tanto se reconoce su tremendo potencial en el aprendizaje, pero también el riesgo de un abuso de Internet. Los alumnos aprenden más cuando son sometidos a una enseñanza interactiva que cuando son meros espectadores en la enseñanza presencial clásica. El nuevo equipamiento facilita las clases colaborativas y las innovaciones tecnológicas, y permite expandir la productividad de un modo insospechado. Sin embargo, la presencia del profesor es irreemplazable, no sólo porque sigue siendo el núcleo central de la clase, sino también porque es el conducto mediante el cual la universidad cumple su labor formativa.

Por otra parte, la educación a distancia o enseñanza virtual es percibida como una alternativa para crecer sin incurrir en sustanciales costos de inversión. El paso de la brick university a la click university abre ciertamente un campo enorme para ampliar el acceso al conocimiento a grandes contingentes de estudiantes y a un costo relativamente bajo. Sin embargo, se corre el riesgo de afectar la calidad del proceso de aprendizaje y depreciar el capital acumulado en prestigio y reputación institucional cuando se pone excesivo hincapié en las ventajas económicas y poco cuidado en las metodologías de enseñanza.

Un campo para el uso de las nuevas tecnologías, que goza actualmente de entusiasta acogida, es su aplicación en los procesos 
administrativos. Las ganancias en eficiencia pueden ser gigantescas. Así, por ejemplo, una aplicación concreta es el empleo de software computacional para el uso de salas de clases. Este consiste en un programa al cual se alimenta de información sobre necesidades y atributos requeridos por los docentes. Luego, mediante un proceso iterativo, va asignando salas, esto es, ocupando espacios hasta agotar las disponibilidades. Este cruce eficiente entre oferta y demanda tiene como corolario un mayor control sobre la inversión en espacio físico, la cual se realiza sólo en tanto se detectan excesos de demanda y congestión.

La presencia de las tecnologías de información aplicadas al campo de la educación superior plantea la pregunta acerca de cómo las instituciones deben organizarse internamente para abordar su desarrollo. La respuesta, en general, es centralizar en un ente que planifique su uso corporativo, goce de cierta autonomía financiera y mantenga estrecho contacto con los potenciales usuarios, los docentes y los administradores universitarios. La visión opuesta de descentralizar estas decisiones tiene el defecto de duplicar esfuerzos con el riesgo de sobreinvertir; además, impide que la universidad negocie mejores condiciones económicas con los proveedores de los programas y equipos.

\section{Respuesta a la globalización y sus efectos}

La globalización trae el mundo a la universidad o, dicho al revés, la integra más allá de sus fronteras para insertarla en ese entorno global. Este fenómeno transforma a la docencia e investigación en commodities transables. Ambas pueden desarrollarse ahora en un ámbito que va más allá del espacio físico de la sala de clase o el laboratorio. Esta apertura tiene ventajas y reparos. Ya hemos dicho que la docencia puede llegar a un mayor número de estudiantes, es una forma eficiente de crecer o, bien, los propios estudiantes tienen la opción de recibir docencia extramuros sin salir de la universidad. Los riesgos van por el lado de la calidad de lo que se ofrece o recibe. 
El laboratorio virtual también presenta un lado positivo al crear redes de investigadores con el consiguiente intercambio de experiencias y trabajo conjunto. La principal amenaza es un deterioro del sentido de comunidad: los académicos pueden sentirse más parte de la comunidad científica internacional -lo que, por una parte, parece bien- perdiendo, por otra, al mismo tiempo, su sentido de pertenencia a la universidad que los acoge y los financia.

En este contexto, es evidente que el desafío es diseñar esquemas que permitan proteger el sentido de comunidad, y es allí donde la gestión a un nivel superior es un factor crucial en el éxito de la tarea. Las universidades deben reconocer las ventajas de la globalización como una oportunidad para mejorar, pero arbitrando medidas para proteger su identidad institucional. Los seminarios y talleres de discusión de profesores, los premios de reconocimiento a la docencia de calidad, los beneficios anexos a los contratos laborales, las políticas de inducción y acogida son todas medidas que buscan mantener la fidelidad institucional.

Por otra parte, la globalización en el plano estudiantil tiene un sentido positivo, porque el intercambio de experiencias de estudiantes que pueden desarrollar parte de los estudios en universidades extranjeras es un complemento valioso en su aprendizaje. Para los estudiantes que pasan un semestre o trimestre en otro país es la oportunidad para conocer otros métodos de estudio y nuevas experiencias de vida personal, lo que ocurre, a su vez, con aquéllos que conviven con alumnos foráneos, quienes también enriquecen su proceso formativo. Las universidades han comprendido la relevancia de estas experiencias, creando departamentos o unidades centrales que se encargan de gestionar convenios, particularmente los referidos a programas de doble titulación, y también orientar a los alumnos que desean viajar al extranjero. 


\section{Restricción de fondos públicos}

Otra característica de estos tiempos es la reducción de los fondos públicos destinados a la educación superior. Los persistentes y agudos déficit fiscales han obligado a los gobiernos a limitar los gastos y destinar los recursos a ciertas áreas prioritarias, afectando los aportes fiscales a esta modalidad educacional.

Cualesquiera sean las estructuras de gobierno, lo cierto es que las universidades han debido tomar medidas encaminadas a reemplazar las fuentes de financiamiento estatales. Este ha sido un campo propicio para la gestión estratégica, ineludible, pero también complejo. La tendencia en Chile, desde que se inicia este proceso a comienzos de los ochenta, ha consistido tanto en un incremento de aranceles de matrícula como en un aumento en las vacantes. No hay dudas de que la presión fiscal ha estimulado un crecimiento en la oferta de cupos, en muchos casos de carácter inorgánico, es decir, sin un respaldo adecuado en mayores recursos invertidos con el obvio deterioro en la calidad de la enseñanza. El menor aporte global del Estado ha ido paralelo a un cambio en la composición del gasto. Han crecido los fondos concursables, lo que ha obligado a las universidades a crear instancias para preparar y presentar adecuadamente proyectos de desarrollo académico. Asimismo, la existencia de leyes nuevas para incentivar los aportes privados -la ley de donaciones, por ejemplo- ha sido otra posibilidad de allegar recursos que ha estimulado la imaginación y obligado a fortalecer la capacidad de gestión.

\section{Evaluación de calidad (accountability)}

Las exigencias de acreditación de competencias en el campo de la docencia de pre y posgrado, entre programas, y de acreditación institucional para evaluar fortalezas institucionales que garanticen un buen uso de los recursos públicos, son otras variable que han llevado a las instituciones universitarias a diseñar modelos de gestión capaces de conducir adecuadamente estos procesos. Por otra parte, varias universidades han decidido iniciar un camino 
propio para acreditar voluntariamente programas académicos en el extranjero. Pero la calidad no sólo debe apreciarse bajo el prisma de accountability. La calidad es un continuo, es la agregación de piezas en un proceso permanente que envuelve a toda la organización. En efecto, la calificación académica es hoy una herramienta fundamental de acreditación de fortalezas. Este suele ser, en general, un proceso llevado a cabo por las propias facultades, que organizan equipos de gestión en su interior para conducir apropiadamente la evaluación de desempeño, sin requerir del apoyo directo de las autoridades centrales. Ello no siempre conduce a resultados efectivos y obliga a elaborar regulaciones internas que muchas veces son fuente de tensión y conflicto.

Sobra referirse a la importancia de la evaluación de productividad y la medición de clima laboral en el estamento administrativo, con el mismo propósito de crear condiciones para un trabajo eficiente de apoyo a la gestión académica.

\section{Universidad-empresa}

La vinculación de la universidad con la empresa privada es uno de los canales de contacto social mediante el cual la universidad pone sus recursos humanos, de carácter científico-tecnológico, al servicio del país y recibe de ella no sólo aportes económicos, sino también experiencias que enriquecen los programas académicos. Desde luego, es una oportunidad para ir creando la motivación entre los estudiantes para desarrollar empresas, es decir, fomentar el espíritu emprendedor a través de cursos para tales fines.

Este paulatino acercamiento con la empresa genera variados cambios en la organización. Por cierto, supone valorar la investigación en una perspectiva amplia, ya que su vinculación con el medio productivo será una fuente valiosa de transformación tecnológica y, por ende, de soluciones eficaces en el plano económico y social. Esto implica coordinar esfuerzos y, por lo tanto, planificar actividades mediante una gestión eficaz, que evite el riesgo de que 
la eventual mayor rentabilidad para los investigadores de contar con aportes de empresas privadas postergue y deteriore la investigación en ciencias básicas. Asimismo, estas iniciativas pueden fomentar la innovación y dar origen a patentes de invención. Es necesario, en consecuencia, poner atención sobre la propiedad del nuevo conocimiento y articular esfuerzos para internalizar estos subproductos en la cadena investigador-universidad.

\section{Conclusiones}

No es claro aún cómo será la universidad del futuro, cuando por universidad entendemos aquella visión que arranca desde la Edad Media y que se consolida con el modelo clásico de una institución generadora de bienes públicos, que forma personas y crea nuevo conocimiento. La misma que hemos tenido en mente al analizar las transformaciones en la gestión en un mundo donde el vértigo del cambio deja poco espacio para la reflexión y la pausa. El nuevo ambiente es, por cierto, más apremiante y hostil y la fuerza de la competencia amenaza por erosionar las bases de la universidad tradicional. Recordemos la visión catastrófica de Peter Drucker: "Las universidades no sobrevivirán. El futuro está fuera de la sala de clases tradicional".

Pero, aún sin caer en fundamentalismos, los riesgos de reaccionar mal y tarde son evidentes: por una parte, existe el peligro de que la universidad se transforme meramente en una fábrica que organiza sus recursos según fines estrictamente económicos. Es la pregunta que se hace P. Gumpart ${ }^{11}$ : ¿ha tomado la educación superior funciones principalmente económicas, abandonando su mandato institucional de desempeñarse en el campo educacional, pero también cumpliendo funciones sociales y políticas? Pero también está el riesgo de la autocomplacencia, de pensar que no

11 Gumpart, Patricia (2001). Built to serve: the enduring legacy of public education. En: Altbach, P., Gumport, J. y Johnstone, B. (ed.) In Defense of American Higher Education, The Johns Hopkins University Press. 
hay amenazas y que bastan la tradición y el prestigio atesorado para responder a este entorno abundante en dificultades.

Nuestro análisis ha mostrado las características de una universidad que se organiza internamente para abordar el actual contexto histórico, sin perder de vista su misión fundacional, y que sabe reaccionar proactivamente frente a los desafíos y tendencias, transformando las amenazas en oportunidades de desarrollo. Ello requiere de elecciones estratégicas claras, eficaz coordinación interna y mucho liderazgo en la conducción. 